\title{
Complex Electron Transfer Pathway at a Microelectrode Captured by in situ Nanospectroscopy
}

\author{
Thomas Touzalin, Suzanne Joiret, Emmanuel Maisonhaute*, Ivan T. Lucas* \\ Sorbonne Universités, UPMC Univ Paris 06, UMR 8235, Laboratoire Interfaces et Systèmes Electrochimiques, F-75005 Pa- \\ ris, France.e-mail: ivan.lucas@upmc.fr ; emmanuel.maisonhaute@upmc.fr.
}

\begin{abstract}
In the quest for analytical tools which enable the characterization of materials at the nanoscale and under the condition of their operation (in situ, operando), the emerging Tip-Enhanced Raman Spectroscopy (TERS) now enters the spotlight. We demonstrate in this work that a TERS tip can be functionalized and partially insulated to be used as a microelectrode enabling Electrochemical Substrate Enhanced Raman Spectroscopy (EC-SERS) at a single hotspot. This "SERS at a tip" experiment enables to capture the electrochemical transformation of a molecular layer self-assembled on a tapered gold microelectrode. The proposed set-up and protocol opens new perspectives in the characterization and development of complex redox architectures for molecular devices.
\end{abstract}

The elaboration of new functional nanomaterials and the understanding of complex (bio) interfaces requires the development of analytical tools which can operate at the nanoscale with a sufficient sensitivity and under the condition of operation of these materials (in situ). Among the emerging techniques enabling such capabilities, vibrational nanospectroscopy like TipEnhanced Raman (TERS) stands apart since it does not require ultra-high vacuum conditions, unlike electron or X-Ray microscopies. In TERS, the apex of a sharp gold or silver tip is illuminated with a visible laser and brought in contact with the sample to analyze. The plasmon resonance triggered at the apex of the tip provides a strong localized electric field (the so-called hot spot) which accounts for the great enhancement of the scattered signal at the tip. ${ }^{1-3}$ The extraction of TERS signatures of compounds/systems with very weak Raman scattering, i.e. selfassembled monolayers (SAMs), nanoparticles or nanowires/tubes is therefore possible and mostly achievable experimentally in a so-called "gap mode" configuration, where the compound under scrutiny is sandwiched between the TERS tip and a gold or silver substrate (terraces or islands) for improved enhancement and acute spatial resolution.

Albeit the implementation of TERS in liquid represents a challenging task because of the difficulty to maintain the delicate optical coupling between the tip and the excitation laser and also to ensure an efficient collection of the scattered signal at the tip, a few pioneering works have recently emerged demonstrating compositional analysis and imaging at the nanoscale in aqueous or organic solvents ${ }^{4-7}$. TERS under electrochemical conditions (EC-TERS) has been also considered and recently demonstrated on model systems chemi- or physisorbed on polarizable substrates. ${ }^{8-11}$ Detection and identification of reaction intermediates by TERS can now be envisaged to decipher complex reaction schemes involving multi-electron transfer to molecular species.

However, the signal fluctuations observed in TERS when scrutinizing only a few molecules under the tip can challenge the real-time quantification of surface processes, although theses variations can also provide important information regarding the local microenvironment of individual molecules. ${ }^{11}$ Besides, in an EC-TERS set-up, other complications may arise from ohmic losses across large polarized substrates. These latter drastically affect the temporal resolution of the electrochemical processes $^{12,13}$ and minimize the benefits of time-resolved TERS experiments. Similar questions may arise in electrochemical Surface-Enhanced Raman Spectroscopy (SERS) for which the signal is collected and averaged on a square micrometer area possibly on an inhomogeneous substrate. ${ }^{10}$ Finally, the close proximity of the tip with the polarized substrate may interfere locally with the electric field, potentially affecting the electrochemical response.

In this work, we introduce an alternative set-up using a partially insulated TERS-active tapered gold electrode directly functionalized with the compound of interest ${ }^{14}$ and immersed into the electrolyte ${ }^{15}$. Our set-up can therefore be assimilated to a single hot spot (particle ${ }^{16}$ ) SERS experiment but implemented on a tapered tip used as a microelectrode. Recently, Pienpinijtham et al. proposed such combination using a gold tip modified by p-mercaptobenzoic acid and p-aminothiophenol to measure a local $\mathrm{pH} .{ }^{17}$ With the proposed design, the spatial resolution of compositional analysis is lost since no functionalized surface is scanned by the tip, but the number of molecules which contribute to the measured Raman signal is larger than in the junction tip/substrate of a classical TERS experiment, providing stable and intense Raman signatures. In addition, the electrode size is reduced to micrometric dimensions, so that the ohmic drop complications vanish and the temporal resolution is improved. For the sake of clarity, the EC-SERS at a tip set-up we introduce here will be denominated as EC-Tip-SERS in the following.

We propose to demonstrate the strong potential of this analytical set-up by studying the electrochemical (EC) reduction of a 4-nitrothiophenol (4-NTP) monolayer. Self-Assembled Monolayers (SAMs) of 4-NTP have been proposed as anchorage 
points for protein immobilization, which can be easily switched from bio-inert to active, depending on the potential applied ${ }^{18}$, i.e. of their redox state. The literature on the ECtransformations of 4-NTP using either advanced electrochemical techniques ${ }^{18-21}$ or EC-SERS ${ }^{22-24}$ abounds, but still the mechanism of its transformation is poorly understood. One identified limitation is the photo induced dimerization of 4-NTP monolayers as revealed by TERS measurements in the air. ${ }^{25-27}$ Implementation of TERS in liquids limits the occurrence of such photochemical processes. ${ }^{9,28}$

By using the Tip-SERS configuration, strong Raman signature of a 4-nitrothiophenol SAM in an aqueous electrolyte can be extracted, providing new insights in its electrochemical reduction mechanism. This protocol could be routinely applied to the characterization of other molecular architectures with redox properties.

\section{EXPERIMENTAL SECTION}

Reagents and Solutions. 4-nitrothiophenol (4-NTP) and 4aminothiophenol (4-ATP) were analytical grade, obtained from Sigma-Aldrich and used without further purification. Absolute ethanol, hydrochloric acid (37\%) and sulfuric acid (96\%) were purchased from VWR and used as received. Water was bi-distilled. Gold wires $(250 \mu \mathrm{m}$ diameter $)$ were purchased from MaTecK (Germany).

Electrochemical measurements. Gold sphere microelectrodes were prepared by melting/quenching a $250 \mu \mathrm{m}$ diameter gold wire in a glass capillary. ${ }^{29}$ Electrodes were functionalized by prolonged immersion ( 3 hours) into a $10^{-4} \mathrm{M}$ ethanolic solution of 4-NTP or 4-aminothiophenol (4-ATP). Physisorbed species were removed by thorough washing with ethanol, and by $3 \times 30$ minutes successive baths in absolute ethanol. Electrodes were then dried in an argon flow. Electrochemical measurements were performed using an Autolab (MetrOhm) potentiostat, model PGSTAT100N, in a three electrode configuration. Gold electrodes stand as working electrodes, a large area platinum grid was used as an auxiliary electrode and a Saturated Calomel Electrode (SCE) as a reference electrode. Electrolytes were deoxygenated by argon bubbling. Their area was obtained by recording cyclic voltammograms of ferrocene solution (5.4 $\mathrm{mM}+0.20 \mathrm{M}$ tetrabutylammonium perchlorate in acetonitrile): the anodic peak was plotted versus the square root of the sweep rate (ranging from 0.1 to $1 \mathrm{~V} \mathrm{~s}^{-1}$ ) using the Randles-Sevcik equation; the diffusion coefficient of ferrocene under these conditions is $2.43 \times 10^{-9} \mathrm{~m}^{2} \mathrm{~s}^{-1}$.

Ex situ TERS mapping: Large and flat $100 \mathrm{~nm}$ thick gold substrates (non-SERS active) were prepared by vacuum deposition on a mica substrate, flame annealed prior to use and functionalized with 4-NTP using the protocol previously described. Tapered gold TERS probes were prepared by electrochemical etching of $250 \mu \mathrm{m}$ diameter gold wire in a $1: 1(\mathrm{~V}: \mathrm{V})$ concentrated $\mathrm{HCl} /$ Ethanol solution as previously described ${ }^{6,30}$ (see Figure S6). Tips were mounted on a Raman (Horiba Labram Evolution HR800, Japan) coupled with a scanning tunneling microscope (AIST/ Omegascope, USA) equipped with a Mitutoyo air objective $(100 x$, N.A. $=0.7)$ mounted on a piezo stage and positioned on the side. A $632.8 \mathrm{~nm}$ linearly polarized HeNe Laser, used as the Raman excitation light source, was focused on the apex of the tip through the objective lens.

Electrochemical Tip-SERS measurements. The above mentioned TERS active tapered gold probes were partially insulated with polyethylene glue to be used as Tip-SERS electrodes and were then functionalized with 4-NTP using the protocol described above. For EC-Tip-SERS measurements, the
STM/Raman system previously described was equipped with a $3 \mathrm{~mm}$ working distance Olympus water immersion objective $(40 \mathrm{x}$, N.A. $=0.8)$ positioned vertically. The functionalized electrode was slightly bent to be brought to the focal point of the objective while still maintaining the polarization of the vertical excitation light source along the shaft of the tip for maximum enhancement (see Figure 4a). In our set-up, the functionalized tip stands as the working electrode, a platinum wire as the auxiliary electrode and a silver wire as a pseudo-reference electrode shifting all the measured potentials of $+208 \mathrm{mV}+/-20 \mathrm{mV} v s$ the Saturated Calomel Electrode (SCE). The immersion objective and the functionalized tip were immersed in a home-made electrochemical cell filled with a $50 \mathrm{mM} \mathrm{H}_{2} \mathrm{SO}_{4}$ aqueous electrolyte. The solution was not deoxygenated.

Reference compounds: spectra of the 4-ATP powder as well as a 4-ATP modified TERS tip were collected at $\lambda=632.8 \mathrm{~nm}$ using a microRaman system (Horiba, LabRam Evolution HR800) and are presented as supporting information (Figure S9).

\section{RESULTS AND DISCUSSION}

Electroactivity of 4-NTP SAMs. The electrochemical response of a 4-NTP SAM was first studied onto a gold sphere microelectrode (area: $0.87 \mathrm{~mm}^{2}$ ) into $50 \mathrm{mM} \mathrm{H}_{2} \mathrm{SO}_{4}$. A first cyclic voltammetry experiment $(\mathrm{CV})$ performed at a scan rate of $100 \mathrm{mV} \mathrm{s}^{-1}$, starting from $+450 \mathrm{mV}$ down to $-550 \mathrm{mV} v \mathrm{~s}$ SCE, shows a chemically irreversible reduction peak at $-265 \mathrm{mV}$ (see inset in Figure 1). This peak disappears during the next cycle, indicating that the entire 4-NTP layer can be effectively and irreversibly reduced, provided that the vertex potential is negative enough. A second CV experiment onto a fresh 4-NTP SAM for which the potential is reversed at higher potential $(-250 \mathrm{mV})$ is presented in Figure 1.

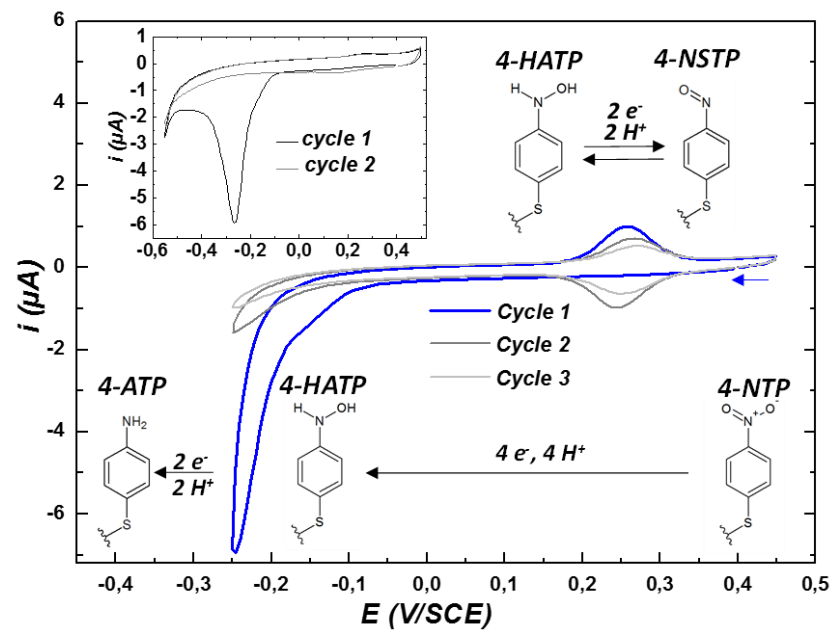

Figure 1. Cyclic voltammetry (CV) response of a 4-Nitrothiophenol functionalized gold sphere electrode in $\mathrm{H}_{2} \mathrm{SO}_{4} 50 \mathrm{mM}$ (scan rate $100 \mathrm{mV} . \mathrm{s}^{-1}$, reference electrode: $\mathrm{SCE}$ ). Main figure: partial reduction of 4-NTP into 4-HATP below $-100 \mathrm{mV} v \mathrm{~s}$ SCE and its reversible oxidation into 4-NSTP at $241 \mathrm{mV} v s$ SCE; Insert: full irreversible reduction of 4-NTP into 4-ATP at potential as low as $-600 \mathrm{mV}$ vs SCE.

In contrast, a reversible system centered on $+241 \mathrm{mV}$ appears in addition to the above mentioned reduction wave. These results are in agreement with the literature. ${ }^{19-21,31,32}$ The reduction peak at $-265 \mathrm{mV}$ corresponds to the reduction of the $\mathrm{NO}_{2}$ function of 4-NTP. However, while the reduction into 4-aminothiophenol (4-ATP) is complete through a $6 \mathrm{e}^{-}-6 \mathrm{H}^{+}$process for ver- 
tex potentials more negative than $-300 \mathrm{mV}$, it may be only partial for higher vertex potentials. In this case 4-hydroxyaminothiophenol (4-HATP) can be generated through a $4 \mathrm{e}^{-}-4 \mathrm{H}^{+}$process. 4-HATP can be oxidized at $+241 \mathrm{mV}$ into 4-nitrosothiophenol (4-NSTP) through a $2 \mathrm{e}^{-}-2 \mathrm{H}^{+}$process, while oxidation of 4-ATP is not observed in the potential range considered in this study. $^{28}$

When scanning the potential backward, 4-NSTP is reduced back to 4-HATP. The reversible peak centered on $+241 \mathrm{mV}$ therefore corresponds to the reversible $2 \mathrm{e}^{-}$process transformation of 4-HATP to 4-NSTP (see main CV in Figure 1). During the two following CVs, the intensity of this reversible peak diminishes indicating that either 4-HATP or remaining 4-NTP are progressively irreversibly reduced into 4-ATP.

This first set of results indicates that the electrochemical transformation process around $-265 \mathrm{mV} v s$ SCE is rather complex since two different redox processes $\left(4 \mathrm{e}^{-}\right.$and $\left.6 \mathrm{e}^{-}\right)$translate into a single broad reduction peak.

Study of the irreversible reduction of 4-NTP. To confirm that the concomitant $6 \mathrm{e}^{-}$and $4 \mathrm{e}^{-}$reduction processes producing 4-ATP and 4-HATP from 4-NTP may occur at potentials as high as $-150 \mathrm{mV} v s \mathrm{SCE}$, quantitative electrochemical investigations were carried out using the same gold sphere electrode.

The electrode surface was reconditioned by flame annealing prior any new experiment. The surface coverage of 4-NTP on the electrode was first determined by integration of the irreversible peak between -90 and $-475 \mathrm{mV}$ assuming a six electron and proton exchange. With this assumption, the coverage was estimated to be $7.73 \times 10^{-6} \mathrm{~mol} \mathrm{~m}^{-2}$ which is slightly higher but consistent with previous results reported under similar conditions. $^{19,31}$

The conversion rate of 4-NTP into 4-HATP ( $v s$ 4-ATP) as a function of the number of $\mathrm{CV}$ scans (up to 10 cycles, scan rate: $100 \mathrm{mV} \mathrm{s}^{-1}$ ) was then estimated for four different vertex potentials $\left(E_{\text {vertex }}=-150,-200,-250\right.$ and $\left.-300 \mathrm{mV}\right)$. The coverage of the 4-NSTP/4-HATP system was obtained for each cycle by integration of the reversible peak at $+241 \mathrm{mV}$ (Figure 2 and Figure S1).

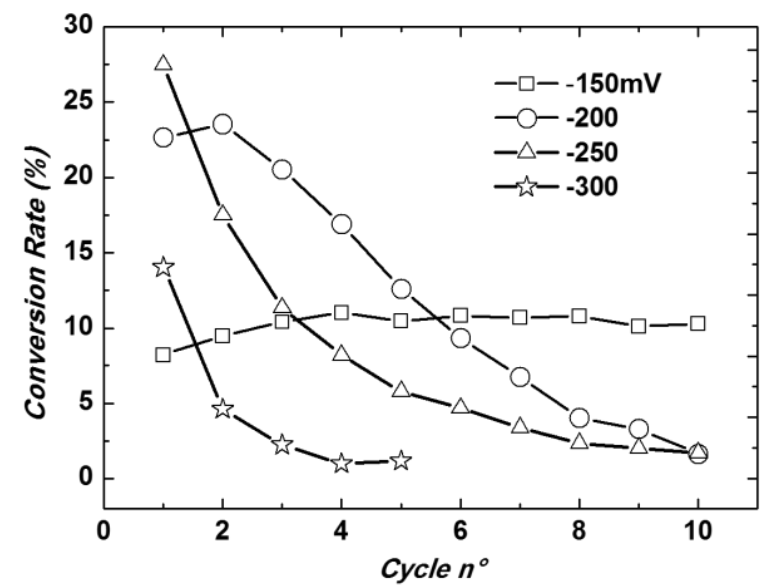

Figure 2. Conversion rates of 4-NTP to 4-HATP extracted from the charge integration of the reversible peak at $+241 \mathrm{mV}$ vs SCE on successive CVs (up to 10). Four different experiment were performed with various potential range, starting from $+450 \mathrm{mV}$ down to, $-200,-250,-300$ and $-350 \mathrm{mV} v s$ SCE respectively.

For the first ten CVs run between $+450 \mathrm{mV}$ and $-150 \mathrm{mV}$, the conversion rate slightly increases to reach a plateau (10\%), indicating that 4-NTP is only partially reduced to 4-HATP with possibly no further reduction to 4-ATP. For the second experi- ment with the potential limits set to $-200 \mathrm{mV}$, a higher conversion rate $(23.5 \%$ of the initial SAM) is observed during the second cycle but decays afterwards, indicating that 4-HATP (and 4-NTP) are progressively irreversibly reduced into 4-ATP at such potential. The maximum conversion rate, $27.5 \%$, of $4-$ NTP to 4-HATP could be observed during the first CV of the third experiment (limit set at $-250 \mathrm{mV}$ ) but it then decreased abruptly. Finally, the fourth experiment (limit set at $-300 \mathrm{mV}$ ) shows similarly a drastic decay from the first cycle, but with a maximum conversion rate of $14 \%$ only. From this set of data, it appears clear that the 4 and 6 electron reduction processes take places concomitantly, the irreversible conversion of 4-NTP to 4-ATP occurring preferentially over the conversion to 4-HATP. Extra data on the reduction of 4-NTP during a chronoamperometric sequence are presented in the supplementary information section and support this conclusion (see Figure S2).
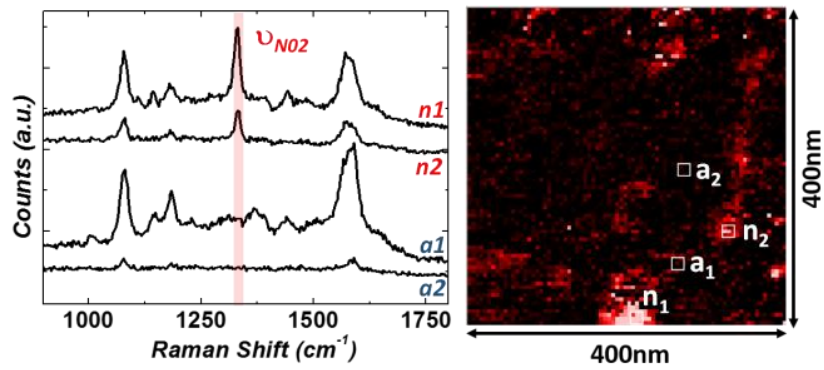

Figure 3. Uncomplete and inhomogeneous reduction (300 s at $300 \mathrm{mV} / \mathrm{SCE}$ ) of a 4-NTP self-assembled monolayer on a flat gold substrate as revealed by ex situ STM-TERS. Selected spectra ( 9 pixel average) from the TERS map: $n_{1}$ and $n_{2}$ correspond to spectra dominated by the 4-NTP bands, $a_{1}$ and $a_{2}$ are dominated by 4-ATP bands. $400 \times 400 \mathrm{~nm}^{2}, 80 \times 80$ pixels TERS mapping of the $\mathrm{NO}_{2}$ band intensity at $1335 \mathrm{~cm}^{-1}$. Laser power $500 \mu \mathrm{W}$, acquisition time $200 \mathrm{~ms}$, tunneling current: $200 \mathrm{pA}$, bias: $100 \mathrm{mV}$.

TERS characterization of 4-NTP electrochemical transformation. To push further the understanding of the reduction process of 4-NTP SAMs, TERS measurements were implemented. A 4-NTP layer was self-assembled on a large flat goldcoated electrode and electroreduced at $-300 \mathrm{mV} v s$ SCE for 5 $\min$ in the above-mentioned electrolyte and then rinsed and dried for compositional analysis (see Figure 3). Ex situ TERS spectra collected on several area of the prepolarized surface, confirm the presence of 4-ATP (Figure 3: $a_{1} \& a_{2}$ ) as previously suggested by the $\mathrm{CV}$ experiment but also reveals the signature of 4-NTP (Figure 3: $n_{1} \& n_{2}$ ). A $400 \times 400 \mathrm{~nm}^{2}$ TERS mapping of the $\mathrm{NO}_{2}$ symmetric stretching band intensity at $1335 \mathrm{~cm}^{-1}$ $\left(\mathrm{U}_{\mathrm{NO} 2}\right)$ reveals the presence of many residues of non-reduced 4NTP at the surface of the polarized gold electrode (red and white pixels), although the full SAM is expected to be reduced at such low potential, based on the previous analysis on the gold sphere electrode (a detailed analysis can be found as the supplemental information in Figure S3 and S4).

On such large metallized substrate, the reduction of 4-NTP can thus be only partial and also inhomogeneous over the surface. As mentioned earlier, ohmic losses and the spatial dispersion of RC charging time constants may affect the effective potential and the electron transfer across the electrode surface. Also, Kolb et al. demonstrated that onto Au(111) surfaces, 4NTP SAMs present disordered and ordered domains. ${ }^{31}$ It may be thus expected that reduction occurs at different rates and at different potentials in the disordered areas versus at the edge and/or inside the organized domains. Another set of data presented as the supplemental information (Figure S5) shows the 
complete reduction of a 4-NTP SAM on a similar substrate after only 30s, highlighting the importance to control the EC-TERS substrate conductivity and homogeneity.

In situ SERS on a functionalized TERS tip. To improve the reliability of in situ analyses, electrochemical Tip-SERS measurements were conducted in the following using a functionalized 4-NTP tapered TERS microelectrode.

a)

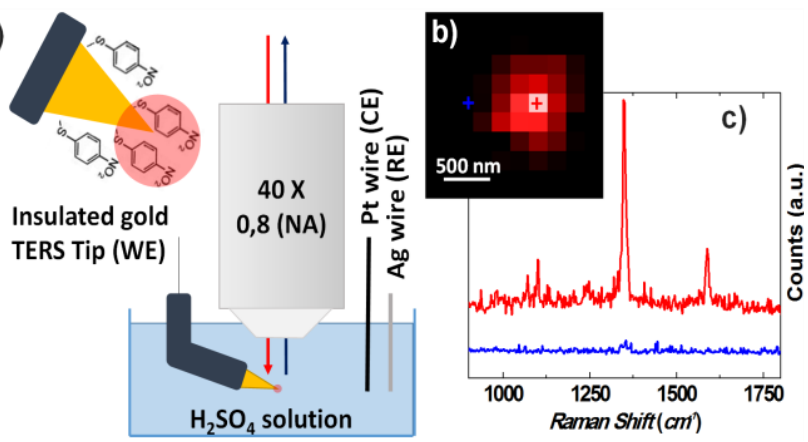

Figure 4. a) Depiction of the electrochemical Tip-SERS set-up including a three electrode cell: a SERS active gold working microelectrode (WE) functionalized by 4-NTP, a platinum counter electrode (CE), a silver wire pseudo-reference electrode (RE) and an immersion objective with high numerical aperture, all immersed in $\mathrm{a} \mathrm{H}_{2} \mathrm{SO}_{4} 50 \mathrm{mM}$ solution, b) $2 \times 2 \mu \mathrm{m}^{2} \mathrm{XY}$ objective map obtained by integration of the peak intensity (with baseline correction) at $1335 \mathrm{~cm}^{-1}$ revealing the location of the "hot spot" on the tip. c) In situ Raman spectra collected at the exact hot spot location (red: "SERS") and slightly away (blue). Laser power $250 \mu \mathrm{W}$, acquisition time $200 \mathrm{~ms}$.

The set-up as depicted on Figure 4a, uses a 40x water immersion objective with high numerical aperture (N.A. $=0.8$ ) and a $3 \mathrm{~mm}$ working distance through which both the excitation laser beam $(\lambda=632.8 \mathrm{~nm})$ and the scattered light from the tip are fed and collected. The use of such objective reported for SERS but never for TERS nor Tip-SERS experiments ${ }^{33}$, enables despite its low magnification a high Raman signal collection efficiency. Its high working distance allows a gold tip mounted on a side holder to reach the focal point of the objective, when bent with a slight angle (between 10-30 ${ }^{\circ}$ ). The objective / gold tip system is immersed in an electrochemical cell filled with a $50 \mathrm{mM}$ $\mathrm{H}_{2} \mathrm{SO}_{4}$ solution. The tip is partially insulated to reduce its electroactive surface. As for ultramicroelectrodes (UME), a homogeneous and fast electrochemical response of the electrode can be expected since the ohmic drop, and the spatial dispersion of $R C$ charging time constants across the electrode are considerably reduced. ${ }^{34,35}$

The objective mounted on a piezo stage enables the search for the hot spot, i.e. the point on the tip apex where the scattered intensity is maximum. The resulting $2 \times 2 \mu \mathrm{m}^{2}$ objective map, generated by integration of the Raman band intensity at 1335 $\mathrm{cm}^{-1}$ (integration is performed between 1305 and $1365 \mathrm{~cm}^{-1}$ ), corresponding to the symmetric $\mathrm{NO}_{2}$ vibration of 4-NTP with no electrical polarization of the tip is presented on Figure $4 \mathrm{~b}$. Two spectra collected in $0.2 \mathrm{~s}$ at the exact hot spot (SERS spectrum of 4-NTP) and slightly away are also presented (Figure 4c, larger SERS maps collected on a functionalized tip can be found in SI in Figure S7). These latter reveal the very localized position of the hot spot, i.e. $0.5 \mu \mathrm{m}$ although imaged through a $3 \mathrm{~mm}$ layer of electrolyte and with a low magnification objective. As a comparison, the hot spot imaged with a 100x air ob- jective $($ N.A. $=0.7)$ through a few hundred micrometers of hexadecane is around $1 \mu \mathrm{m}$ as found by Touzalin et al. in a previous work. ${ }^{6}$ Moreover, when illuminating the gold tip under the same conditions but with a $532 \mathrm{~nm}$ green laser, i.e. an excitation light source which does not induce the plasmon resonance of gold, only a very weak chemical signature could be extracted, confirming the SERS activity of the functionalized tip at $632.8 \mathrm{~nm}$.

Potential dependent Tip-SERS signature. In the following, the SERS signature of the 4-NTP functionalized Au electrode was recorded after one $\mathrm{CV}$ cycle (scan rate $100 \mathrm{mVs}^{-1}$, starting potential $+250 \mathrm{mV} v \mathrm{Ag}$ ). The lower vertex potential was set to $-200 \mathrm{mV}$ down to $-500 \mathrm{mV}$ vs $\mathrm{Ag}$ (i.e. to $-300 \mathrm{mV}$ vs $\mathrm{SCE}$ ) for the successive "CV and Tip-SERS" sequences, to capture the electrochemical transformation of 4-NTP. Each spectrum was acquired at a fixed "relaxation" potential $E_{\mathrm{SERS}}=-200$ $\mathrm{mV}$ vs $\mathrm{Ag}(+8 \mathrm{mV}+/-20 \mathrm{mV}$ vs SCE), where no electrochemical reaction is expected, with a $100 \mu \mathrm{W}$ laser power and a $5 \mathrm{~s}$ integration time. Note that, the laser power was kept minimal to avoid the photochemical reduction of densely packed 4-NTP into the dimeric form dimercaptoazobenzene (DMAB), often reported in the air or in alkaline medium. ${ }^{25,26,36-39}$

After the first CV down to $E_{\text {vertex }}=-200 \mathrm{mV} v s \mathrm{Ag}(+8 \mathrm{mV}$ $v s$ SCE), the TERS signature of $4-\mathrm{NTP}^{40}$ was obtained as depicted on Figure 5, with prominent features at 1078 (with a shoulder at 1050), 1100, 1335 and $1577 \mathrm{~cm}^{-1}$ (corresponding vibrations are described in Table 1) confirming that no reaction takes place at such potential.

For the following CVs (2 to 3 ) which explored lower potentials, some alteration of the 4-NTP Tip-SERS signature are observed, i.e. a relative decrease of the band intensities at 1050 , 1100 and 1335 (with a slight blue shift) and $1577 \mathrm{~cm}^{-1}$ as compared to the band at $1078 \mathrm{~cm}^{-1}$. However, no spectral features specific to 4-HATP compound $\left(1230 \mathrm{~cm}^{-1}\right)$ could be detected as suggested by the gold sphere electrode experiment. After the $4^{\text {th }}$ and $5^{\text {th }}$ cycles which extended the time electrode was polarized at $-450 \mathrm{mV}$ vs $\mathrm{Ag}$, the $1335 \mathrm{~cm}^{-1}$ band intensity is lowered by a factor of two, the shoulder at $1050 \mathrm{~cm}^{-1}$ has disappeared and a new band emerges at $1593 \mathrm{~cm}^{-1}$ and progressively grows in intensity (extra bands also at 400,625, $950 \mathrm{~cm}^{-1}$ not assigned).

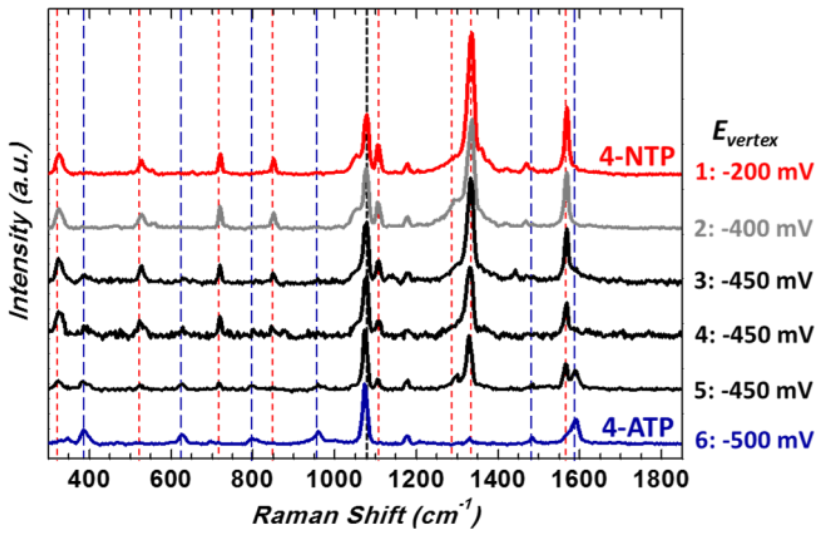

Figure 5. Electrochemical Tip-SERS spectra of a 4-NTP functionalized gold electrode in $\mathrm{H}_{2} \mathrm{SO}_{4} 50 \mathrm{mM}$. Each spectrum was acquired at ESERS $=-200 \mathrm{mV} v s \mathrm{Ag}(+8 \mathrm{mV}$ vs SCE) with a 100 $\mu \mathrm{W}$ laser power and a $5 \mathrm{~s}$ integration time after a $\mathrm{CV}$ (scan rate: $\left.100 \mathrm{mV} . \mathrm{s}^{-1}\right)$ covering the range $\left[+250 \mathrm{mV}-E_{\text {vertex }}\right]$. $E_{\text {vertex }}$ was set to $-200 \mathrm{mV}$ down to $-500 \mathrm{mV}$ vs $\mathrm{Ag}$ ( +8 to $-308 \mathrm{mV} v s \mathrm{SCE}$ ). All spectra were normalized relatively to the $1080 \mathrm{~cm}^{-1}$ band intensity and offset for clarity. Red and blue dashed lines highlight the band associated to 4-NTP and 4-ATP respectively. 
The $6^{\text {th }}$ sequence, where the electrode was polarized at potentials as low as $-500 \mathrm{mV} v \mathrm{Ag}$, shows the complete disappearance of the band at $1335 \mathrm{~cm}^{-1}$ and the dominance of the band at $1593 \mathrm{~cm}^{-1}\left(+400,625\right.$ and $\left.950 \mathrm{~cm}^{-1}\right)$ which characterizes the formation of $4-\mathrm{ATP}^{41}$ (the detailed band intensity variation with the number of cycle is given in Figure S8). Note that no peak appears in the region $1400-1500 \mathrm{~cm}^{-1}$, characteristic of the dimeric form of 4-NTP or of derived reduction products, so that in this study there is no plasmon assisted photochemical transformation. ${ }^{26}$

The decrease in intensity of the symmetric stretching vibration $v\left(\mathrm{NO}_{2}\right)$ from spectra (1) to (6) attests of the disappearance of 4-NTP upon reduction at -200/-250 $\mathrm{mV}$ vs SCE. However, no obvious signatures of the expected vibration $v(\mathrm{~N}(-\mathrm{OH}))$ for 4-HATP 22 were detected although the conversion rate of 4-NTP to 4-HATP was found maximum on the gold sphere electrode at such potential. Note that upon positive polarization of the electrode, the vibration $\mathrm{v}(\mathrm{N}=\mathrm{O})$ of $4-\mathrm{NSTP}^{42}$ could not be detected either. The somehow limited conversion rate to 4-HATP (27,5\% at max) and its possible weak Raman signature could explain why it has been not observed here and neither reported in any prior study. ${ }^{22-24}$ As proposed on the gold sphere electrode and confirmed here on Tip-SERS spectrum (5) the conversion to 4-ATP occurs during repeated polarization at $-258 \mathrm{mV} v \mathrm{~s}$ SCE and quickly dominates the compositional signature. This is further confirmed by the fair reproduction of the spectra (2) to (5) using a linear combination of spectra (1) and (6) (Figure $\mathrm{S} 10)$. After the $6^{\text {th }}$ sequence extending the potential limit to $308 \mathrm{mV}$ vs SCE during a few seconds only, the 4-NTP signature has totally disappeared, indicating that the whole SAM has been reduced on the Tip-SERS electrode. This behavior is fully consistent with the one observed on the gold sphere microelectrode. This set of results on the 4-NTP reduction thus establishes our approach as a robust and effective way to capture in situ transformation processes at a microelectrode.

\begin{tabular}{|c|c|c|c|}
\hline Molecule & Vibration & $\begin{array}{c}\text { Raman } \\
\text { shift (experimental) }\end{array}$ & Reference \\
\hline \multirow{5}{*}{ 4-NTP } & $\mathrm{C}-\mathrm{H}$ bending & $1079 \mathrm{~m}(1080, \mathrm{~m})$ & \multirow{5}{*}{ [36] } \\
\hline & $\mathrm{C}-\mathrm{H}$ bending & $1100 \mathrm{~s}(1110, \mathrm{~s})$ & \\
\hline & $\mathrm{C}-\mathrm{H}$ bending & $1181 \mathrm{~m}(1180, \mathrm{w})$ & \\
\hline & $\begin{array}{l}\mathrm{NO}_{2} \text { symmetric } \\
\text { stretching }\end{array}$ & 1335 vs $(1335$, vs $)$ & \\
\hline & C-C stretching & 1577 s $(1570, s)$ & \\
\hline \multirow{3}{*}{ 4-ATP } & C-S stretching & 1089 vs $(1080, s)$ & \multirow{3}{*}{ [37] } \\
\hline & $\mathrm{C}-\mathrm{H}$ bending & $1173 \mathrm{~m}(1180, \mathrm{~m})$ & \\
\hline & C-C stretching & 1593 s $(1588, s)$ & \\
\hline \multirow{2}{*}{ 4-NSTP } & $\mathrm{N}=\mathrm{O}$ stretching & $\begin{array}{c}\text { 1488-1523 (not } \\
\text { observed) }\end{array}$ & \multirow[t]{2}{*}{ [38] } \\
\hline & $\begin{array}{c}\text { C-N stretching + ring } \\
\text { vibrations }\end{array}$ & $\begin{array}{c}1318+1342 \text { (not } \\
\text { observed) }\end{array}$ & \\
\hline 4-HATP & & 1230 (not observed) & [18] \\
\hline
\end{tabular}

Table 1. Main characteristic Raman vibrations for 4NTP/ATP/NSTP/HATP compounds as described in the literature. The dimeric form DMAB shows bands around $1400 \mathrm{~cm}^{-1}$ (not reported here). Intensities: vs: very strong, s: strong, m: medium, w: weak.

\section{CONCLUSIONS}

In this work, we have demonstrated single hot spot electrochemical SERS using functionalized TERS tips and high numerical immersion objective (EC-Tip-SERS), by probing in situ the electrochemical transformation of a 4-NTP monolayer self-assembled on a tapered gold microelectrode. EC-TipSERS experiments are a great alternative to EC-SERS experi- ments for which the spectral signature are averaged on microscopic area, with possibly non-uniform electron transfer and also to EC-TERS experiments probing a limited number of molecules in the tip/substrate junction. Also, in this EC-Tip-SERS experiment using minimal power laser, no photo degradation of the 4-NTP SAM (dimerization) was observed to interfere with the reduction process of 4-NTP as often reported in the literature.

The Raman signal extraction upon exploration of reducing potentials enabled to track the progressive transformation of the nitro group of 4-NTP to amino (4-ATP) and revealed that the intermediate $4 \mathrm{e}^{-}$reduction of the nitro group of 4-NTP to hydroxylamine (4-HATP) competes with the $6 \mathrm{e}^{-}$reduction to amine (4-ATP) at potential as high as $-150 \mathrm{mV}$ vs SCE where this later is not expected to occur. The lower the potential, the more dominant $6 \mathrm{e}^{-}$conversion over $4 \mathrm{e}^{-}$. Surprisingly, Tip-SERS spectra collected at more and more reducing potentials could be reproduced by the combination of 4-NTP and 4-ATP spectra only. Raman signatures of the intermediates 4-HATP and 4NSTP never reported before could not be detected either with Tip-SERS here, confirming the competition between the two electrochemical paths. The low conversion rate of 4-NTP to 4HATP/4-NSTP (27.5\% at max), the fast decay of their surface concentration and the possible weak Raman signature of 4HATP/4-NSTP could explain their absence in the Tip-SERS spectra.

The successful in situ characterization of 4-NTP monolayer supports the efficiency of EC-Tip-SERS analyses as baseline characterization for redox architectures which the mechanism of transformation is still to be determined. The hybrid tips developed herein, i.e. TERS ready ultra-microelectrodes also pave the way to time resolved EC-TERS experiments. ${ }^{13}$

\section{AUTHOR INFORMATION}

\section{Corresponding Author}

Ivan T. Lucas (ivan.lucas@upmc.fr) and Emmanuel Maisonhaute (emmanuel.maisonhaute@upmc.fr).

\section{ORCID}

Ivan T. Lucas: 0000-0001-8930-0437

Emmanuel Maisonhaute: 0000-0001-6337-7436

\section{ACKNOWLEDGMENT}

The authors would like to thank Labex Michem (Sorbonne Universités, ANR-11-IDEX-0004-02), the Europe Council (FP7People-2013-CIG program: "ENSOM" Project, ID: 618643") and the île de France county (Dim Nano-K) for their financial support in the acquisition of the nano/microRaman system Horiba/AIST.

\section{ASSOCIATED CONTENT}

\section{Supporting Information}

Extra material can be found as supporting information: detailed CV experiments on the gold sphere electrode, the conversion rate at a gold sphere electrode during chronoamperometric steps, additional ex situ TERS characterization of 4-NTP electrochemical transformation, the EC-Tip-SERS probe characteristics, the 4-NTP and 4-ATP main Raman band intensity evolution during the $\mathrm{CV}$ experiment shown in Figure 5, the comparison of the Raman spectra of 4-ATP obtained on a powder and with the functionalized TERS tip, and the combination of the Tip-SERS spectra of 4-NTP and 4-ATP which allow to reproduce fairly the intermediate spectra obtained during the EC-TipSERS experiment. 


\section{REFERENCES}

(1) Stöckle, R. M.; Suh, Y. D.; Deckert, V.; Zenobi, R. Chem. Phys. Lett. 2000, 318, 131-136.

(2) Wang, Y.; Rogers, E. I.; Compton, R. G. J. Electroanal. Chem. 2010 648, 15-19.

(3) Kazemi-Zanjani, N.; Vedraine, S.; Lagugne-Labarthet, F. Opt. Express 2013, 21, 25271-25276.

(4) Schmid, T.; Yeo, B.-S.; Leong, G.; Stadler, J.; Zenobi, R. J. Raman Spectrosc. 2009, 40, 1392-1399.

(5) Nakata, A.; Nomoto, T.; Toyota, T.; Fujinami, M. Anal. Sci. 2013, 29, 865-869.

(6) Touzalin, T.; Dauphin, A. L.; Joiret, S.; Lucas, I. T.; Maisonhaute, E. Phys. Chem. Chem. Phys. 2016, 18, 15510-15513.

(7) Martin Sabanes, N.; Driessen, L. M.; Domke, K. F. Anal. Chem. 2016, $88,7108-7114$.

(8) Kurouski, D.; Mattei, M.; Van Duyne, R. P. Nano Lett. 2015, 15, 79567962 .

(9) Zeng, Z. C.; Huang, S. C.; Wu, D. Y.; Meng, L. Y.; Li, M. H.; Huang, T. X.; Zhong, J. H.; Wang, X.; Yang, Z. L.; Ren, B. J. Am. Chem. Soc. 2015, 137, 11928-11931.

(10) Zaleski, S.; Wilson, A. J.; Mattei, M.; Chen, X.; Goubert, G.; Cardinal, M. F.; Willets, K. A.; Van Duyne, R. P. Acc. Chem. Res. 2016, 49, $2023-$ 2030 .

(11) Mattei, M.; Kang, G.; Goubert, G.; Chulhai, D. V.; Schatz, G. C.; Jensen, L.; Van Duyne, R. P. Nano Lett. 2017, 17, 590-596.

(12) Amatore, C.; Maisonhaute, E. Anal. Chem. 2005, 77, 303 A-311 A.

(13) Fortgang, P.; Maisonhaute, E.; Amatore, C.; Delavaux-Nicot, B.; Iehl, J.; Nierengarten, J. F. Angew. Chem. Int. Ed. Engl. 2011, 50, 2364-2367.

(14) El-Khoury, P. Z.; Gong, Y.; Abellan, P.; Arey, B. W.; Joly, A. G.; Hu, D.; Evans, J. E.; Browning, N. D.; Hess, W. P. Nano Lett. 2015, 15, 23852390.

(15) Masson, J. F.; Breault-Turcot, J.; Faid, R.; Poirier-Richard, H. P.; Yockell-Lelievre, H.; Lussier, F.; Spatz, J. P. Anal. Chem. 2014, 86, 8998 9005.

(16) Shi, X.; Coca-Lopez, N.; Janik, J.; Hartschuh, A. Chem. Rev. 2017.

(17) Pienpinijtham, P.; Vantasin, S.; Kitahama, Y.; Ekgasit, S.; Ozaki, Y. J. Phys. Chem. C 2016, 120, 14663-14668.

(18) Mendes, P. M.; Christman, K. L.; Parthasarathy, P.; Schopf, E.; Ouyang, J.; Yang, Y.; Preece, J. A.; Maynard, H. D.; Chen, Y.; Stoddart, J. F. Bioconjug. Chem. 2007, 18, 1919-1923.

(19) Tsutsumi, H.; Furumoto, S.; Morita, M.; Matsuda, Y. J. Colloid Interface Sci. 1995, 171, 505-511.

(20) Médard, C.; Morin, M. J. Electroanal. Chem. 2009, 632, 120-126.

(21) Schwamborn, S.; Stoica, L.; Neugebauer, S.; Reda, T.; Schmidt, H. L.; Schuhmann, W. ChemPhysChem 2009, 10, 1066-1070.

(22) Shindo, H. J. Chem. Soc., Faraday Trans. 1 1986, 82, 45.
(23) Gao, P.; Gosztola, D.; Weaver, M. J. The Journal of Physical Chemistry 1988, 92, 7122-7130.

(24) Chen, X.-J.; Cabello, G.; Wu, D.-Y.; Tian, Z.-Q. J. Photochem. Photobiol., C 2014, 21, 54-80.

(25) Kumar, N.; Stephanidis, B.; Zenobi, R.; Wain, A. J.; Roy, D. Nanoscale 2015, 7, 7133-7137.

(26) Sun, M.; Zhang, Z.; Zheng, H.; Xu, H. Sci Rep 2012, 2, 647.

(27) van Schrojenstein Lantman, E. M.; Deckert-Gaudig, T.; Mank, A. J.; Deckert, V.; Weckhuysen, B. M. Nat. Nanotechnol. 2012, 7, 583-586.

(28) Lukkari, J.; Kleemola, K.; Meretoja, M.; Ollonqvist, T.; Kankare, J. Langmuir 1998, 14, 1705-1715.

(29) Amatore, C.; Maisonhaute, E.; Schollhorn, B.; Wadhawan, J. ChemPhysChem 2007, 8, 1321-1329.

(30) Ren, B.; Picardi, G.; Pettinger, B. Rev. Sci. Instrum. 2004, 75, 837-841. (31) Nielsen, J. U.; Esplandiu, M. J.; Kolb, D. M. Langmuir 2001, 17, 34543459 .

(32) Stratmann, L.; Clausmeyer, J.; Schuhmann, W. ChemPhysChem 2015, $16,3477-3482$.

(33) Zeng, Z.-C.; Hu, S.; Huang, S.-C.; Zhang, Y.-J.; Zhao, W.-X.; Li, J.F.; Jiang, C.; Ren, B. Anal. Chem. 2016, 88, 9381-9385.

(34) Zhou, X. S.; Mao, B. W.; Amatore, C.; Compton, R. G.; Marignier, J. L.; Mostafavi, M.; Nierengarten, J. F.; Maisonhaute, E. Chem. Commun. 2016, 52, 251-263.

(35) Watkins, J. J.; Chen, J.; White, H. S.; Abruña, H. D.; Maisonhaute, E.; Amatore, C. Anal. Chem. 2003, 75, 3962-3971.

(36) Lopez-Ramirez, M. R.; Aranda Ruiz, D.; Avila Ferrer, F. J.; Centeno, S. P.; Arenas, J. F.; Otero, J. C.; Soto, J. J. Phys. Chem. C 2016, 120, 1932219328.

(37) Tabatabaei, M.; Sangar, A.; Kazemi-Zanjani, N.; Torchio, P.; Merlen, A.; Lagugné-Labarthet, F. J. Phys. Chem. C 2013, 117, 14778-14786.

(38) Jiang, R.; Zhang, M.; Qian, S.-L.; Yan, F.; Pei, L.-Q.; Jin, S.; Zhao, L.B.; Wu, D.-Y.; Tian, Z.-Q. J. Phys. Chem. C 2016, 120, 16427-16436. (39) Zhang, Z.; Deckert-Gaudig, T.; Singh, P.; Deckert, V. Chem. Commun. 2015, 51, 3069-3072.

(40) Skadtchenko, B. O.; Aroca, R. Spectrochim. Acta, Part A 2001, 57, 1009-1016.

(41) Osawa, M.; Matsuda, N.; Yoshii, K.; Uchida, I. J. Phys. Chem. 1994, 98, 12702-12707.

(42) Lin-Vien, D.; Colthup, N. B.; Fateley, W. G.; Grasselli, J. G. In The Handbook of Infrared and Raman Characteristic Frequencies of Organic Molecules, Elsevier, Ed.; Academic Press: San Diego, 1991, p 503.

\section{For TOC only}

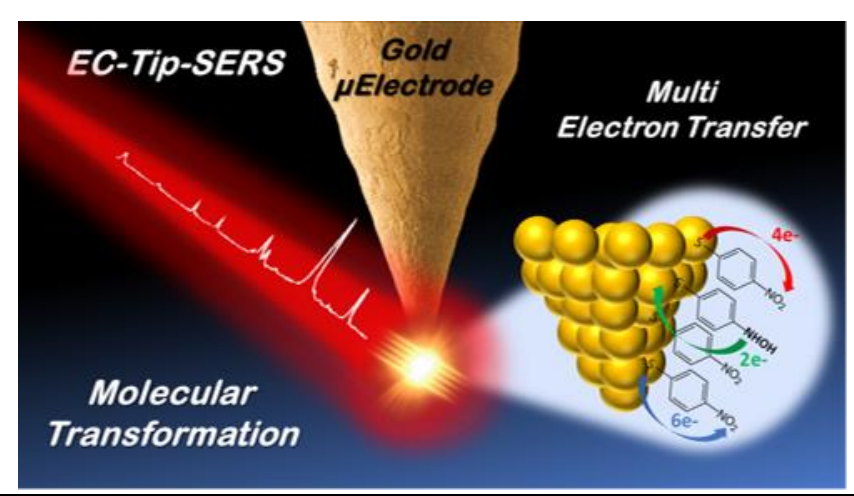

